\title{
Embryonic organoids recapitulate early heart organogenesis
}

Giuliana Rossi ${ }^{1}$, Andrea Boni ${ }^{2}$, Romain Guiet ${ }^{3}$, Mehmet Girgin ${ }^{1}$, Robert G. Kelly ${ }^{4}$, Matthias P. Lutolf ${ }^{1,5, *}$

${ }^{1}$ Laboratory of Stem Cell Bioengineering, Institute of Bioengineering, School of Life Sciences and School of Engineering, École Polytechnique Fédérale de Lausanne (EPFL), Lausanne, 1015, Vaud, Switzerland

${ }^{2}$ Viventis Microscopy Sàrl, EPFL Innovation Park, building C, Lausanne, 1015, Vaud, Switzerland

${ }^{3}$ Faculté des sciences de la vie, Bioimaging and Optics Platform, École Polytechnique Fédérale de Lausanne (EPFL), Bâtiment AI, Station 15, Lausanne, 1015, Vaud, Switzerland

${ }^{4}$ Aix-Marseille Université, CNRS UMR 7288, IBDM, Marseille, France

${ }^{5}$ Institute of Chemical Sciences and Engineering, School of Basic Science, École Polytechnique

Fédérale de Lausanne (EPFL), Lausanne, 1015, Vaud, Switzerland

*Correspondence: matthias.lutolf@epfl.ch

Keywords: Embryonic organoids, cardiogenesis, development, in vitro organogenesis, heart, $3 \mathrm{D}$ cardiac tissue, cardiac organoid. 


\begin{abstract}
Organoids are powerful models for studying tissue development, physiology, and disease. However, current culture systems disrupt the inductive tissue-tissue interactions needed for the complex morphogenetic processes of native organogenesis. Here we show that mouse embryonic stem cells (mESCs) can be coaxed to robustly undergo the fundamental steps of early heart organogenesis with an in vivo-like spatiotemporal fidelity. These axially patterned embryonic organoids support the generation of cardiovascular progenitors, as well as first and second heart field compartments. The cardiac progenitors self-organize into an anterior domain reminiscent of a cardiac crescent before forming a beating cardiac tissue near a putative primitive gut-like tube, from which it is separated by an endocardial-like layer. These findings unveil the surprising morphogenetic potential of mESCs to execute key aspects of organogenesis through the coordinated development of multiple tissues. This platform could be an excellent tool for studying heart development in unprecedented detail and throughput.
\end{abstract}




\section{Introduction}

Stem-cell-derived organoids self-organize into complex structures that mimic aspects of the architecture, cellular composition, and function of tissues found in real organs (Clevers, 2016; Lancaster and Knoblich, 2014; Rossi et al., 2018; Sasai, 2013). While most organoids simulate specific features of adult organs, embryonic organoids can capture key processes that occur during early embryonic development, from the pre-implantation blastocyst (Rivron et al., 2018) to early post-implantation development (Harrison et al., 2017; Shao et al., 2017a, 2017b; Sozen et al., 2018; Zheng et al., 2019) and gastrulation (Beccari et al., 2018; van den Brink et al., 2014). Although embryonic organoids have been shown to mimic many of the morphological and transcriptional hallmarks of the early embryo, their potential to undergo organogenesis has not yet been explored.

The heart is the first organ to form and function in the embryo. Short after gastrulation, heart progenitors are specified, progressively localize anteriorly and organize in a crescentshaped domain (around E7.5), the first cardiac compartment that is morphologically identifiable during development. Cardiogenesis is based on the interaction of two different types of progenitors, namely the first heart field (FHF) and the second heart field (SHF) progenitors, the latter originating from pharyngeal mesoderm (Kelly et al., 2014). The cardiac crescent, mainly formed by FHF progenitors, subsequently rearranges to form a linear heart tube (around E8.0E8.5) which is the first beating structure. Successively, SHF progenitors contribute to heart tube elongation and the heart undergoes looping, ballooning and septation, giving rise to the fourchambered structure typical of adulthood (Harvey, 2002). Heart organogenesis requires cardiac progenitors to interact with surrounding tissues through mechanical interactions and the secretion of cardiac-inducing factors (Miquerol and Kelly, 2013). These involved tissues especially include the endothelium (Brutsaert, 2003; Brutsaert et al., 1998; Narmoneva et al., 2004) and foregut (Hosseini et al., 2017; Kidokoro et al., 2018; Lough and Sugi, 2000; Nascone and Mercola, 1995; Schultheiss et al., 1995; Varner and Taber, 2012).

We hypothesized that due to their embryo-like multi-axial organization and gene expression patterns, mouse gastruloids (Beccari et al., 2018; van den Brink et al., 2014) could offer a suitable template for studying early heart development because they potentially preserve the crucial tissue-tissue interactions required for this organogenesis. Indeed, studying heart organogenesis in such a complex and spatially organized system could allow the modeling of 
developmental events in an embryo-like context, where cardiac cells are naturally exposed to the influence of other tissues.

Here we show that self-organizing mouse embryonic stem cells (mESCs) can capture early heart organogenesis in vitro with a surprising temporal and spatial accuracy. Exposing small ESC aggregates to a cocktail of three cardiogenic factors in gastruloid culture conditions promotes cardiac development in vitro starting from Mesp $^{+}$progenitors, which progressively become restricted to the anterior portion of the gastruloid. Through a combination of light-sheet and confocal microscopy, RNAscope imaging, and FACS, we demonstrate that these embryoids support the formation of $\mathrm{Flkl}^{+}$cardiovascular progenitors, the generation of a vascular-like network, and the formation of progenitors with a first and second heart field identity. Strikingly, we find evidence for the morphogenesis of an anterior cardiac crescent-like domain, which subsequently gives rise to a beating compartment exhibiting $\mathrm{Ca}^{2+}$-handling properties compatible with functional fetal cardiomyocytes. Morphogenesis was established in close spatial proximity to the most anterior portion of a co-developing gut-tube-like structure, which was separated from the cardiac domain by an endocardial-like layer. Therefore, this in vitro model of cardiac organogenesis uniquely captures interactions between embryonic tissues in the context of a spatially organized embryo-like entity. 


\section{Results}

\section{Optimization of culture conditions to promote efficient beating portions in gastruloids}

Gastruloids occasionally formed a beating domain that is exclusively located within their anterior region $(38.5 \pm 29.3 \%$ formed at $168 \mathrm{~h})$ when cultured for $144 \mathrm{~h}$ or longer in N2B27 medium (Fig. 1A). The location and activity of this beating structure suggested that it might correspond to a cardiac primordium. We tested whether well-known cardiogenic factors (Rajala et al., 2011) could increase the frequency of this event by adding basic fibroblast growth factor, ascorbic acid, and vascular endothelial growth factor 165 (VEGF) (Kattman et al., 2006), singly or in combination, and we increased nutrient and growth factor availability through volume optimization and shaking (Fig. 1A, Fig. S1A). In these culture conditions (termed N2B27+++), the frequency of beating gastruloids increased by more than a factor of two $(87.2 \pm 15.6 \%$ formed at 168 h) (Fig. 1A, B and Supplemental Movie 1). We noticed that exposure to cardiogenic factors was most effective when applied in combination and between 96 and $144 \mathrm{~h}$ (Fig. S1A-F), so we kept this protocol for the following experiments. Importantly, culturing in $\mathrm{N} 2 \mathrm{~B} 27+++$ did not alter the polarization of the gastruloids, the extent of their elongation (Fig. S1I-J), nor the timing of the emergence of the beating domains (Fig. 1A, Fig. S1B-D) compared to standard conditions. Staining for Gata4 and cardiac troponin T (cTnT) confirmed that the beating structure was cardiac-like (Fig. 1C).

In cardiomyocytes, physical contraction is coupled to electrical excitation through intracellular changes of $\mathrm{Ca}^{2+}$ (Tyser et al., 2016). To evaluate the functionality of the gastruloid cardiac domain, we thus assessed calcium transients, momentary spikes in voltage, by live gastruloid imaging via light-sheet microscopy (at $168 \mathrm{~h}$ ). Image analysis revealed rhythmic calcium spiking in beating areas with a frequency comparable to beating rates observed in embryos from the crescent stage to the linear heart tube (Tyser et al., 2016) (Fig. 1D-F, Supplemental Movie 2). Drugs interfering with calcium transport, including the 1-type calcium channel blocker nifidepine and the $\beta$-adrenergic agonist isoproterenol, either completely inhibited these transients (Fig. S2A) or caused an increase in spiking frequency (Fig. S2B, C), respectively. These data demonstrate that the cardiac compartments found in late gastruloids (168 h) exhibit $\mathrm{Ca}^{2+}$-handling properties compatible with functional cardiomyocytes. 
A

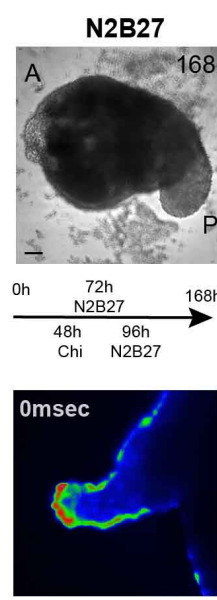

$\mathbf{G}$
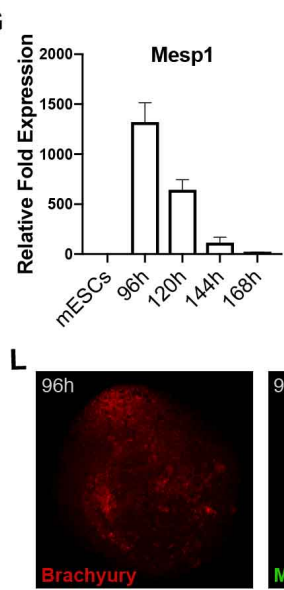

M

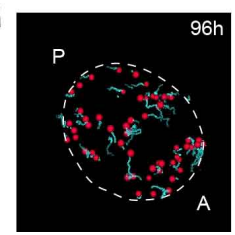

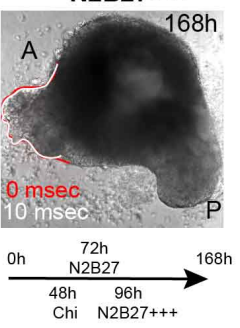

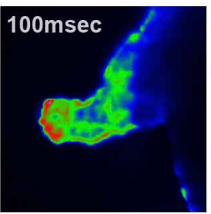

H
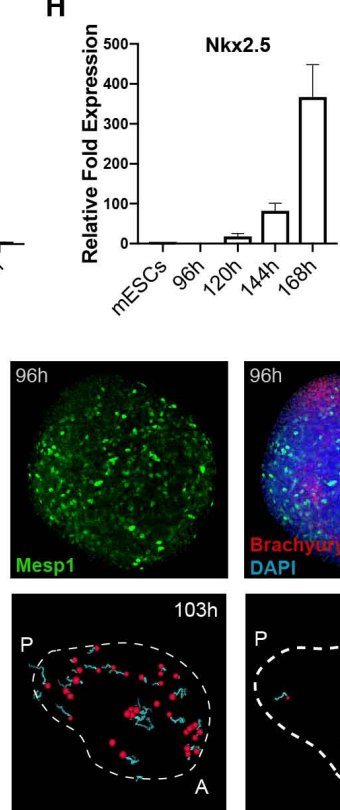

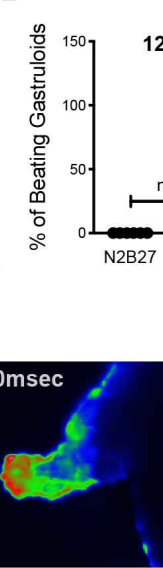

I

$$
\text { I }
$$
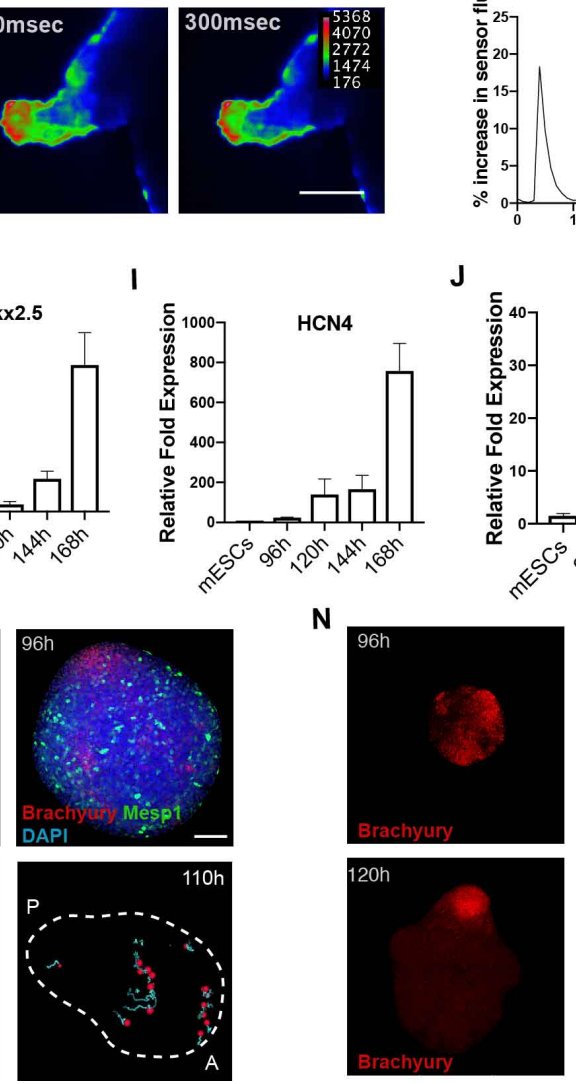

E

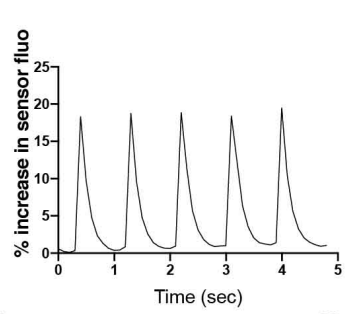

J

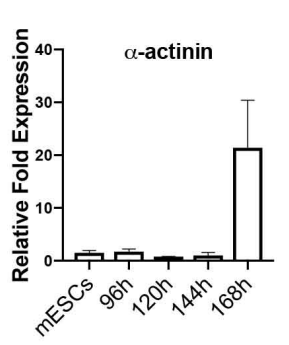

C
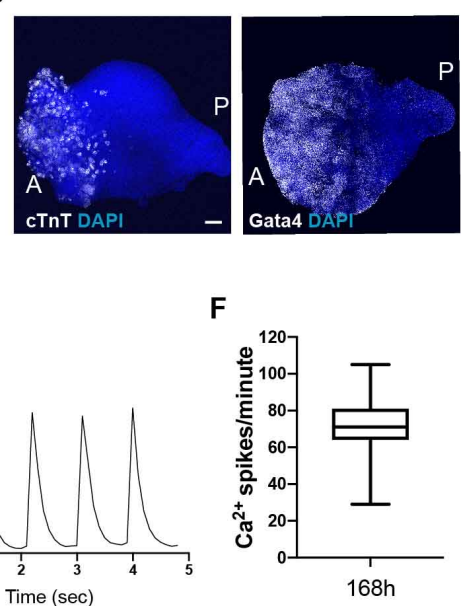

K
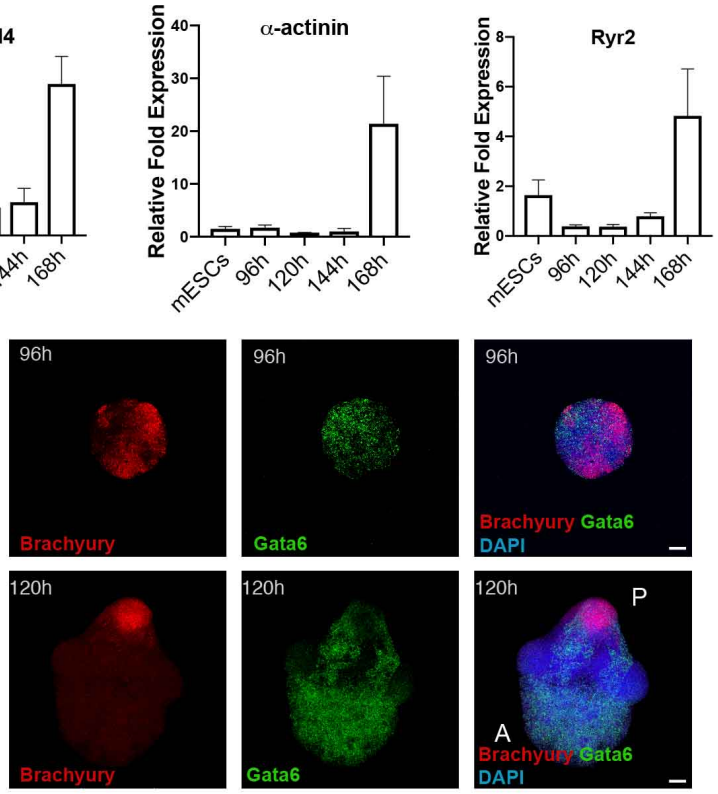

Fig. 1 | Embryonic organoids recapitulate heart organogenesis. A, Gastruloids form a beating portion on their anterior side at $168 \mathrm{~h}$. White and red lines highlight the displacement of the beating domain over $10 \mathrm{msec}$. B, Frequency of beating structures at different time points in $n=6$ independent experiments. C, Immunofluorescence for Gata4 and cTnT on gastruloids at $168 \mathrm{~h}$. D, Calcium imaging E, representative spiking profile and $\mathbf{F}$, spiking frequency of the gastruloid cardiac portion at $168 \mathrm{~h}$ for $n=21$ gastruloids. G$\mathbf{K}$, qPCR gene expression profiles of cardiac genes in gastruloids from 96-168 h. Data are expressed as relative fold expression compared to mESCs in $n=4$ independent experiments. $\mathbf{L}$, Spatial localization of Mesp $1^{+}$cells (stained for GFP) at $96 \mathrm{~h}$ as compared to Brachyury expression. M, Tracking of Mesp $1+$ cells from $96-110 \mathrm{~h}$. Red: tracked cells; light blue: cell tracks forward. N, Spatial localization of Gata6 $^{+}$cells at 96 and $120 \mathrm{~h}$ compared to Brachyury expression. Scale bars, $100 \mu \mathrm{m}$. A: anterior; P: posterior. +++: N2B27 with cardiogenic factors.

\section{The formation of a cardiac domain mimics in vivo heart development}

To understand if the formation of the cardiac portion mimics developmentally relevant processes, we first analyzed the temporal expression of key genes involved in cardiovascular specification (Fig. 1G-K). Similar to what happens during embryonic development (Lescroart et al., 2014; Saga et al., 1996), the first upregulated cardiac gene was Mespl, which was expressed around $96 \mathrm{~h}$ and then rapidly downregulated (Fig. 1G, Fig. S2D, E). Using a Mesp 1- 
GFP reporter ESC line (Bondue et al., 2011) and live light-sheet imaging, we observed that Mesp1 expression started in a mosaic-like manner at $96 \mathrm{~h}$, with Mespl-positive cells first confined to the anterior side before slowly disappearing after $120 \mathrm{~h}$ (Fig. 1M, Supplemental Movie 3). At the same time, and concurrently with the elongation and formation of an anteriorposterior axis, Gata6-expressing cells localized to the anterior side in gastruloids generated from Gata6-Venus ESCs (Freyer et al., 2015), opposite to the pole that was positive for Brachyury (Fig. 1N). Gata6 expression was maintained over time (Fig. S2F, G). From this stage onwards, the early differentiation genes $N k \times 2-5$ and HCN4 were increasingly expressed (Fig. 1H,I), followed by genes marking mature cardiomyocytes ( $\alpha$-actinin, Ryr2) (Fig. 1J, K). This sequence of gene expression shows that gastruloids, stimulated with cardiogenic factors, recapitulate the temporal and spatial gene expression dynamics of cardiac development from the specification of cardiac progenitors to the formation of a beating cardiac structure.

A

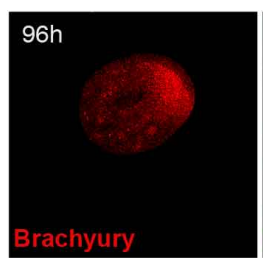

C
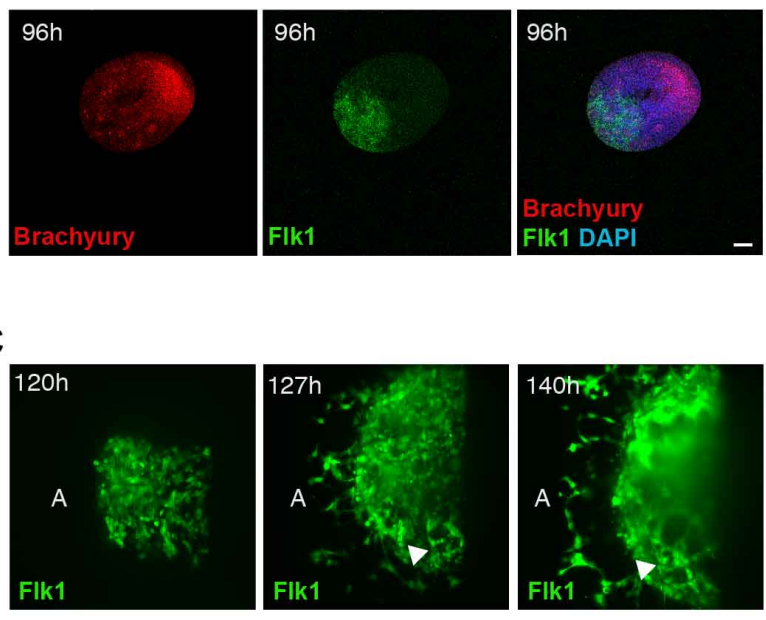

B
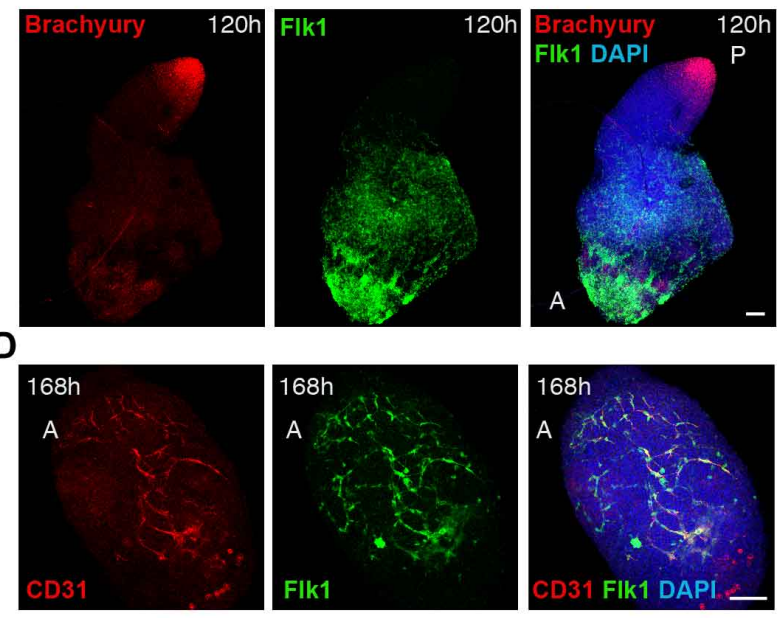

E
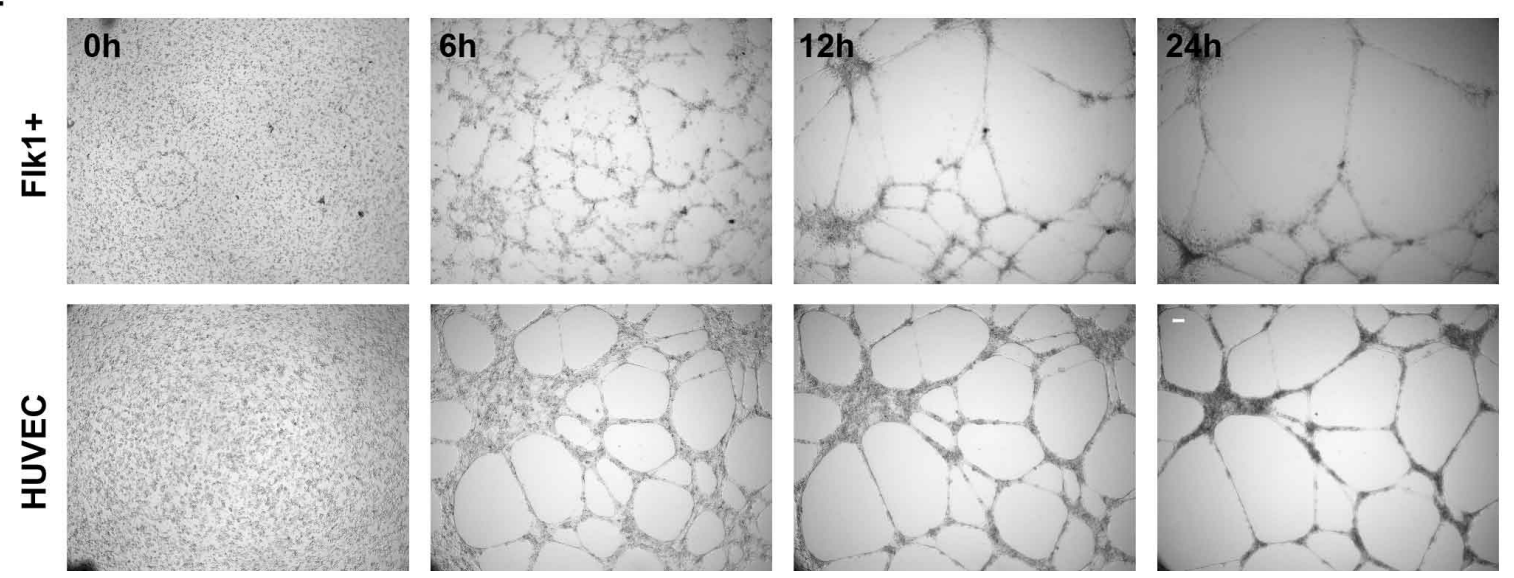

Fig. 2 | Development of a vascular-like network. Spatial localization of $F l k 1^{+}$cells at (A) 96 and (B) $120 \mathrm{~h}$ compared to Brachyury expression. C, Light-sheet live imaging of the anterior portion of Flk1-GFP gastruloids from 120 to $144 \mathrm{~h}$, highlighting the formation of a vascular-like network. D, The Flk1 ${ }^{+}$vascularlike network is positive for CD31. E, Angiogenesis assay showing tube formation in isolated $F l k 1^{+}$cells compared to HUVEC. Scale bars, $100 \mu \mathrm{m}$. A: anterior; P: posterior. 


\section{Co-development of a vascular compartment within the cardiac domain}

During embryonic development, the continuous cross-talk of endothelial cells in the developing heart is a prerequisite for cardiomyocyte maturation, function, and survival (Brutsaert, 2003). For this reason, we tested whether such tissue-tissue interactions could potentially take place in developing gastruloids, focusing on cardiovascular progenitors expressing the well-known marker Flk1 (also known as Kdr or Vegfr2) (Kattman et al., 2006). In 96-h gastruloids derived from a Flk1-GFP reporter ESC line (Jakobsson et al., 2010), Flkl was expressed at the anterior pole opposite to Brachyury (Fig. 2A). Over time, Flkl expression persisted in the anterior portion of the gastruloids (Fig. 2B, Fig. S3A, B), and Flkl-positive cells started to form a vascular-like network of spindle-shaped cells (Fig. 2C, Supplemental Movie 4) that stained positive for the endothelial marker CD31 (Fig. 2D, Fig. S3C). In an in vitro angiogenesis assay, Flk1-positive cells that were isolated by fluorescence-activated cell sorting (FACS) from 168-h gastruloids and plated on Matrigel formed vascular-like networks similar to human umbilical vein endothelial cells (HUVECs) (Fig. 2E, Fig. S3D). Collectively, these results suggest that gastruloids comprise regions that develop into a vascular-like compartment, which is associated with undergoing cardiac development.

\section{In vitro cardiac development entails first and second heart field development}

A key feature of cardiogenesis is a coordinated interaction between two distinct mesodermal progenitor populations: the FHF, which contributes to the left ventricle and part of the atria, and the SHF, which gives rise to the outflow tract, right ventricle, and part of the atria (Harvey, 2002; Miquerol and Kelly, 2013). After migration from the posterior primitive streak, FHF progenitors form the cardiac crescent and early heart tube, located anteriorly. SHF progenitors, originating from cardiopharyngeal mesoderm (Cortes et al., 2018), are characterized by delayed differentiation and are located medially to the crescent, which can then be involved in hearttube elongation. Each of these populations is characterized by a specific pattern of gene expression that we also see in our embryonic organoids; we observed the expression of FHF (Tbx5) and SHF (Tbxl) markers (Fig. 3A-C) in mutually exclusive cell populations, and Isll mostly overlapping with $T b x l$ expression (Fig. 3D). To corroborate the presence of progenitor populations from both heart fields in our organoids, we used a recently published protocol (Andersen et al., 2018) for FACS-isolating SHF progenitors based on the expression of the C$\mathrm{X}-\mathrm{C}$ chemokine receptor type 4 (CXCR4) (Fig. 3E). We observed that Gata $^{+} / \mathrm{CXCR} 4^{+}$cells express higher levels of SHF markers (Tbx1, Isl1, and FGF10), but low or unchanged levels of FHF markers (Nkx2-5, Tbx5, and HCN4) compared to $\mathrm{Gata}^{+}{ }^{/ \mathrm{CXCR}} 4^{-}$, confirming their SHF 
identity (Fig. 3F-K). Together, these data show that late gastruloids contain key cell types that are involved in vivo in first and second heart-field-based cardiogenesis.
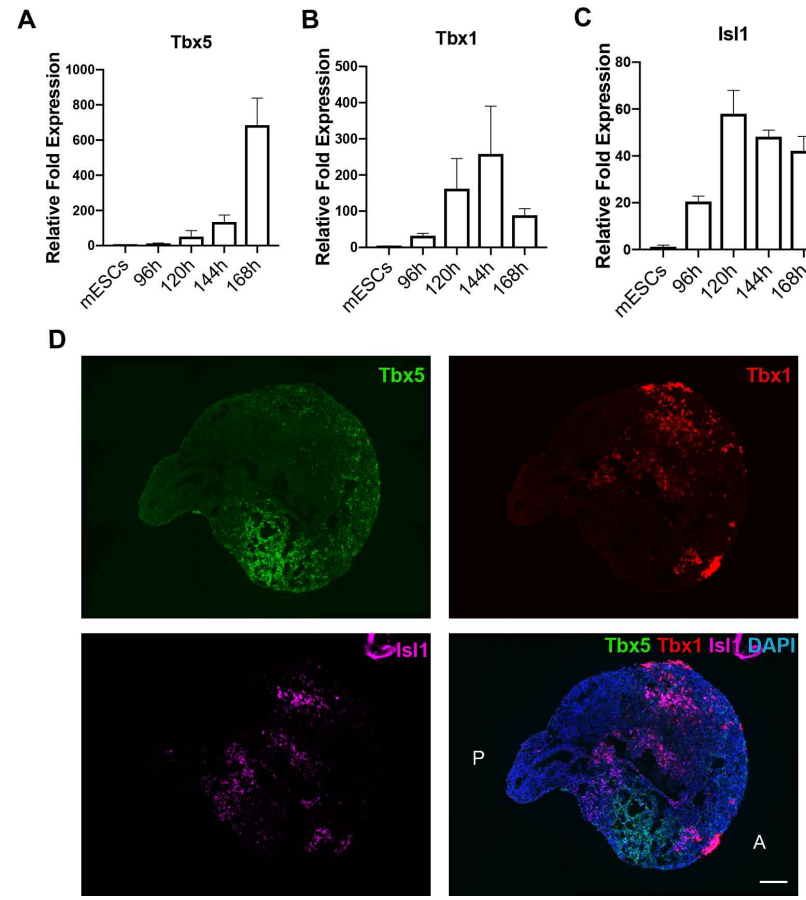
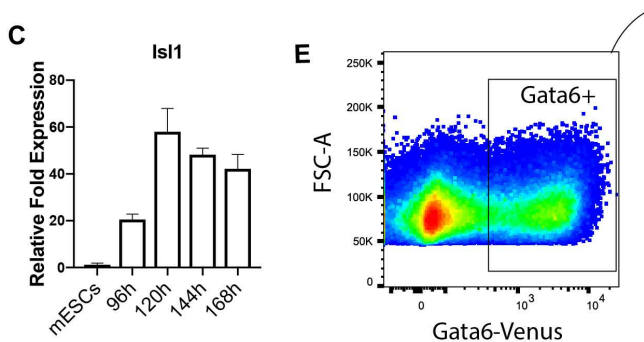

$\mathrm{F}$

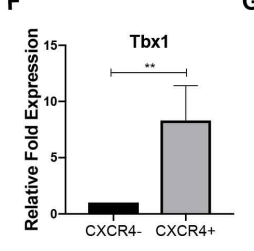

G
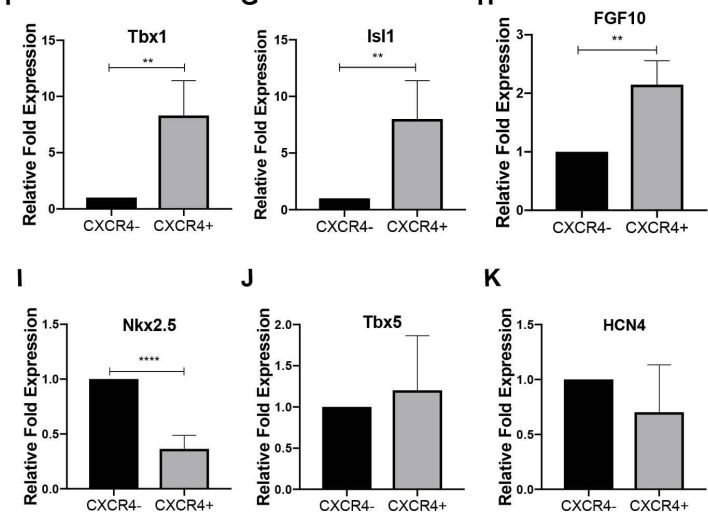

Fig. 3 | Embryonic organoids form a first and second heart field. Expression levels of markers of (A) FHF and $(\mathbf{B}, \mathbf{C})$ SHF in gastruloids from 96 to $168 \mathrm{~h}$. Data are expressed as relative fold expression compared to mESCs in $n=4$ independent experiments. D, RNA-scope showing spatial localization of FHF and SHF domains. E, Representative plot showing the gating strategy used to isolate SHF-enriched progenitors. Expression levels of markers of (F-H) SHF and (I-K) FHF in $\mathrm{Gata}^{+} / \mathrm{CXCR}^{-}$and $\mathrm{Gata}^{+} / \mathrm{CXCR}^{+}$cells isolated from gastruloids at $168 \mathrm{~h}$. Data are expressed as relative fold expression compared to Gata $\sigma^{+} / \mathrm{CXCR}^{-}$cells in $n=4$ independent experiments. Scale bar, $100 \mu \mathrm{m}$. A: anterior; P: posterior.

\section{In vitro cardiac development occurs through establishment of a crescent-like structure}

After their specification in the mouse embryo, cardiac progenitors migrate antero-laterally and progressively fuse at the midline to define the first morphologically identifiable heart structure, the cardiac crescent, which appears around E7.5 (Miquerol and Kelly, 2013). Then, morphogenetic movements associated with foregut closure form a linear heart tube by E8.5 (Miquerol and Kelly, 2013). We explored whether gastruloids stimulated with cardiogenic factors could capture these morphological hallmarks of cardiogenesis. Remarkably, in gastruloids cultured for $144 \mathrm{~h}$ to $168 \mathrm{~h}$, we observed a recapitulation of these events. Around 144 h, cTnT-positive cardiomyocytes were organized in crescent-like domains (Fig. 4A). These further developed into denser crescent-like structures with beating areas, leading to beating epithelial protrusions on the anterior portion of the gastruloids at around $168 \mathrm{~h}$. Similar to mouse embryos (Ivanovitch et al., 2017; Le Garrec et al.; Tyser et al., 2016), these phenotypes could be observed in gastruloids within $24 \mathrm{~h}$, and could be aligned to progressive morphological stages 
of embryonic cardiac development between E7.5 and E8.5 (Fig. 4B). Indeed, a comparative volume analysis of the cTnT-positive gastruloid domains with defined artificial shapes revealed a gradual transition from an almost spherical to a crescent-like shape (144 to $168 \mathrm{~h}$ ) that then became concave (168 h) (Fig. 4C). These results highlight the remarkable capacity of embryonic organoids to promote the spatially and temporally orchestrated morphogenetic processes involved in the early stages of heart development.

A

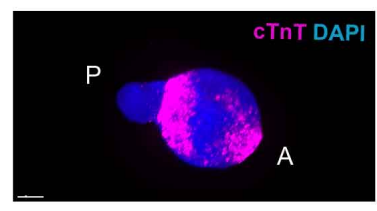

B

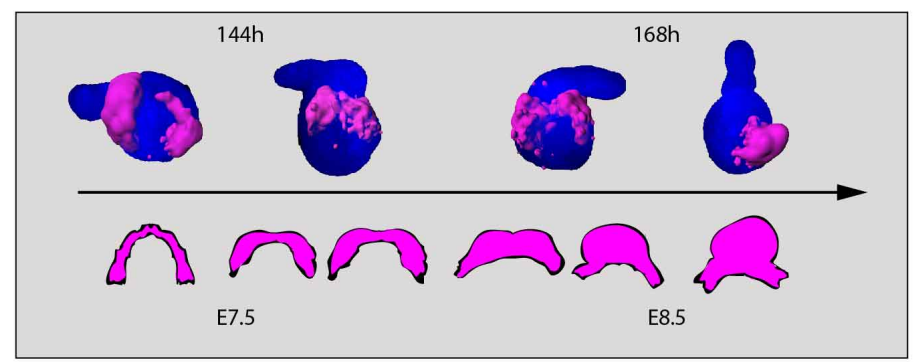

$144 \mathrm{~h}$

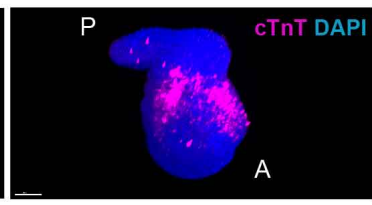

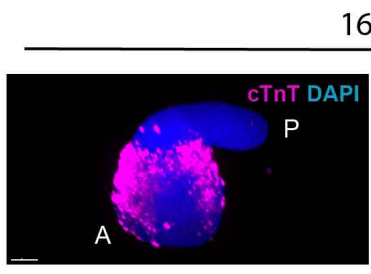

C
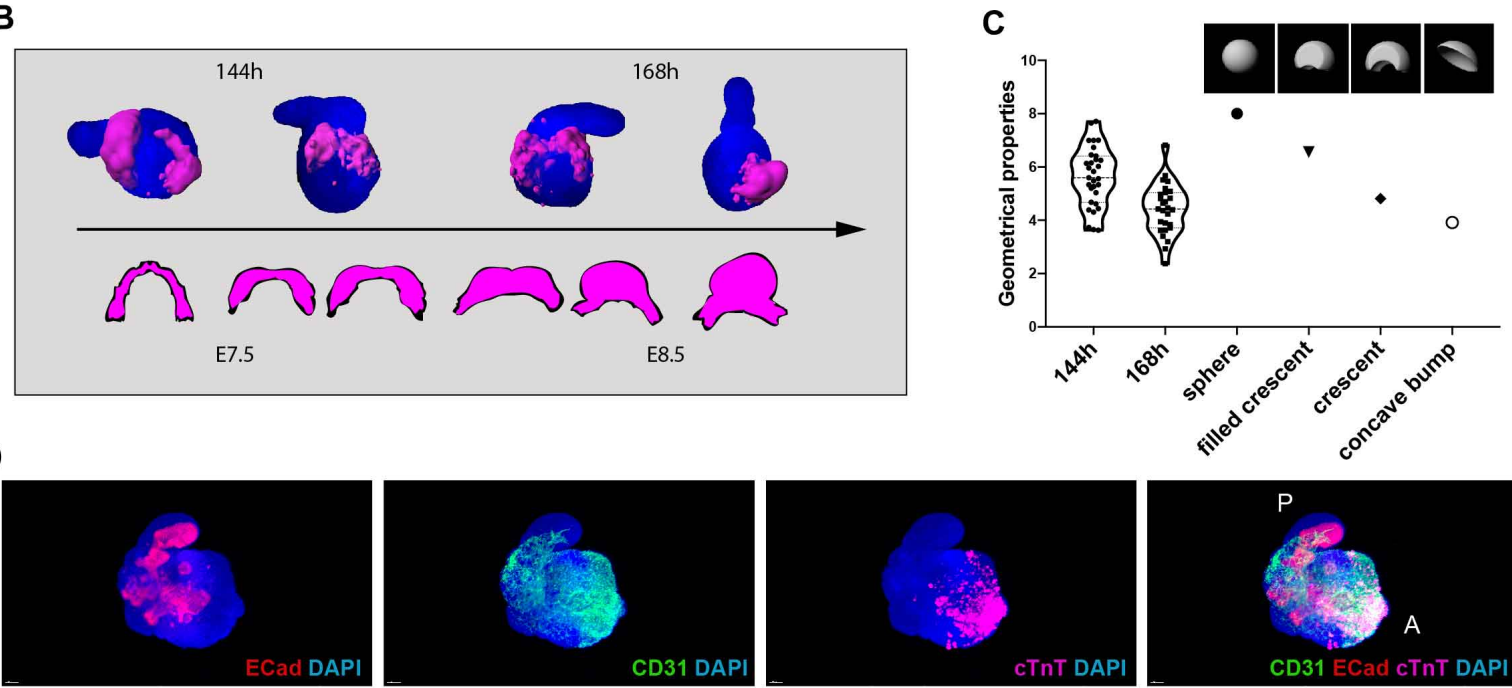

$\mathrm{E}$
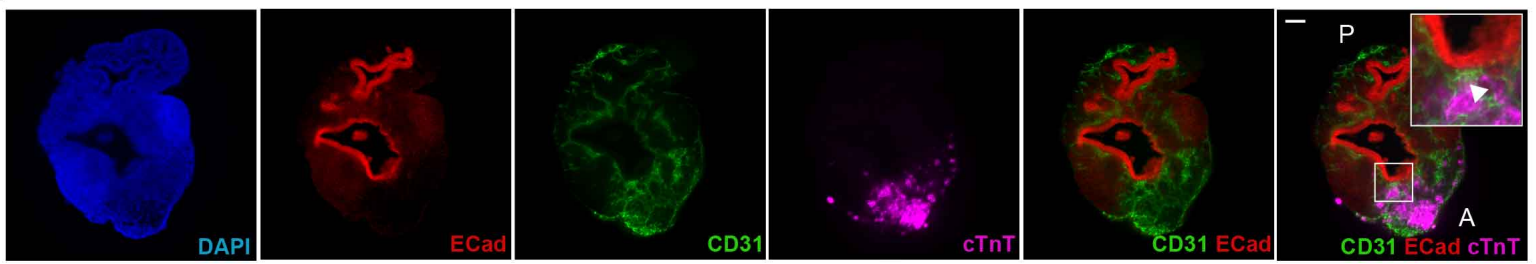

Fig. 4 | Embryonic organoids recapitulate cardiac morphogenesis. A, Light-sheet imaging of cleared gastruloids from 144-168 h show an initial crescent-like domain that is condensed into a beating bud at $168 \mathrm{~h}$. B, Schematic illustrating the comparison between gastruloid stages of cardiac development and embryonic stages from cardiac crescent to linear heart tube. C, Quantification of geometrical properties (spareness) of the gastruloid cardiac domains compared to those of defined artificial shapes, with $n=31$ gastruloids at $144 \mathrm{~h}$ and $n=27$ gastruloids at $168 \mathrm{~h}$. D, E, The cardiac domain is localized near the anterior epithelial gut-tubelike structure, separated by a CD $31^{+}$endocardial layer. Scale bar, $100 \mu \mathrm{m}$. A: anterior; P: posterior. 


\section{In vitro cardiogenesis happens in the context of physiological tissue-tissue interactions}

The presence of a crescent-like structure and its evolution to a coherent beating group of cells led us to test whether this was accompanied by an association between the crescent and anterior endoderm, as is the case in the embryo (Ivanovitch et al., 2017; Lough and Sugi, 2000). Bulk transcriptomics analysis of late gastruloids revealed gene expression patterns (Sox17, Shh, CDX2) that suggested the presence of a primitive gut-tube-like tissue (Beccari et al., 2018). Strikingly, we found that the cTnT-positive cardiac domain in $168 \mathrm{~h}$ gastruloids was exclusively located next to a tube-shaped E-Cadherin-positive epithelial tissue $(n=14 / 14)$, with a CD31positive, putative endocardial-like layer in between (Fig. 4D,E, Supplemental Movie 5). This is reminiscent of the spatial arrangement of anterior structures in the developing embryo (Ivanovitch et al., 2017). 


\section{Discussion}

The heart develops through complex interactions between cardiac progenitors and surrounding tissues (Miquerol and Kelly, 2013). These interactions are crucial for sustaining cardiogenesis but have thus far not been reproduced in vitro. Indeed, numerous in vitro cardiac models, such as engineered heart muscles (Huebsch et al., 2016; Lind et al., 2017; Ma et al., 2015; Mills et al., 2019; Zhao et al., 2019) or cardiac spheroids (Giacomelli et al., 2017; Polonchuk et al., 2017), have been developed, though the in vitro modeling of heart morphogenesis has been out of reach. Our results demonstrate that embryonic organoids can be stimulated to recapitulate in vitro the key steps of early cardiac development that in vivo require polarized interactions between different primordia. These interactions are critical for achieving the architectural and compositional complexity that are absent in conventional organoids (Rossi et al., 2018), but appear to be provided by the multi-axial properties and spatial organization of the different embryonic tissues that are characteristic of gastruloids (Beccari et al., 2018).

We show that embryonic organoids can be stimulated to form a cardiac portion that involves key developmental hallmarks such as the generation and spatial arrangement of early cardiac progenitors and first and second heart field compartmentalization. Of note, precardiac spheroids were previously shown to generate progenitors with FHF and SHF identity, however, these spheroids did not develop an organized structure (Andersen et al., 2018). In contrast, in our model system, these populations emerged in an embryo-like, spatially organized structure. Moreover, the cardiac portion in our system co-developed with a vascular network and an endodermal component, both known to influence cardiac development (Brutsaert, 2003; Lough and Sugi, 2000). Due to the importance of these neighboring developing tissues, previous attempts were performed to derive spheroids composed of both endothelial and cardiac cells (Giacomelli et al., 2017; Polonchuk et al., 2017). However, these systems, based on an aggregation of cells pre-differentiated in 2D, lacked any visible structural organization. Future work will explore whether the morphogenetic processes seen in our system require the presence of spatial complexity (e.g. multi-axial embryonic patterning) and crosstalk with other tissues.

Collectively, our data show that embryonic organoids have the potential for modeling organogenesis. Herein, we have steered the self-organization of small aggregates of mESCs to form a cardiac primordium as a precursor of an embryonic heart. In the future, it will be interesting to study whether cardiac primordia can be further developed and matured, and what type of tissue-tissue interactions are functionally involved in their development. Additionally, 
the principles presented here should be applicable to other organ systems once it is possible to overcome the significant challenge of the limited lifespan of embryonic organoids.

\section{Acknowledgements}

We thank Alfonso Martinez Arias for great support throughout the project. We thank Denis Duboule and Nadia Mercader for useful feedback on the manuscript. We thank members of the Lutolf laboratory for discussions and sharing materials, Cédric Blanpain (ULB) for providing Mesp1-GFP cells, Alexander Medvinsky (MRC Edinburgh) for providing Flk1-GFP cells, Andy Oates (EPFL) and Peter Strnad (Viventis Sàrl) for making light-sheet LS1 microscope available and for technical support, Olivier Burri for EasyXT development, Thierry Laroche for support with Z1 light-sheet imaging, Arne Seitz and other members of Bioimaging and Optics Facility (EPFL) for microscopy support, Jessica Sordet-Dessimoz and Gian-Filippo Mancini from Histology Core Facility for RNAscope, and all personnel of Histology Core Facility, Flow Cytometry Core Facility and Gene Expression Core Facility (EPFL) for their technical support. This work was funded by EPFL.

\section{Author Contributions}

G.R. and M.P.L. conceived the study, designed experiments, analyzed data and wrote the manuscript. G.R. performed the experiments, A.B. performed in vivo light-sheet imaging, R.G. developed the script for the analysis of cardiac structures, M.G. contributed to protocol optimization and data analysis, R.G.K. contributed to study design and data discussion and provided feedback on the manuscript.

\section{Declaration of interests}

A.B. is part of Viventis Microscopy Sàrl that has commercialized the LS1 light-sheet microscope used in this study for time-lapse imaging of gastruloids. The Ecole Polytechnique Fédérale de Lausanne has filed for patent protection on the approach described herein, and M.P.L. and G.R. are named as inventors on those patent applications. 


\section{Materials and Methods}

\section{Cell Culture}

mESCs were cultured at $37^{\circ} \mathrm{C}, 5 \% \mathrm{CO}_{2}$ in DMEM supplemented with $10 \%$ Embryonic Stem Cell qualified FBS (Gibco), NEAA, Sodium Pyruvate, $\beta$-mercaptoethanol, $3 \mu \mathrm{M}$ CHI99201 (Chi), $1 \mu \mathrm{M}$ PD025901 and $0.1 \mu \mathrm{g} \mathrm{ml}{ }^{-1}$ LIF. Gata6-Venus (Freyer et al., 2015), Flk1-GFP (Jakobsson et al., 2010), and Mesp1-GFP (Bondue et al., 2011) cells were cultured on gelatincoated tissue culture flasks; Soxl-GFP::Brachyury-mCherry (Deluz et al., 2016) cells on tissue-culture flasks without coating. If not differently specified, Sox1-GFP::Brachyury$m$ Cherry cells were used for our experiments. HUVECs were cultured in EGM-2 medium (Lonza). All cells were routinely tested for Mycoplasma with Mycoalert mycoplasma detection kit (Lonza) or by PCR.

\section{Gastruloid culture}

Gastruloids were generated as previously described (Baillie-Johnson et al., 2015). Briefly, 300$700 \mathrm{mESCs}$ were plated in 40 $\mu 1$ N2B27 in 96-well Clear Round Bottom Ultra-Low Attachment Microplates (7007, Corning). After 48 h, 150 $\mu$ l of N2B27 containing $3 \mu \mathrm{M}$ Chi were added to each well. After $72 \mathrm{~h}$, medium was changed with N2B27. Starting from $96 \mathrm{~h}$, the protocol was optimized as described in Fig. S1A. At 96 h, gastruloids were transferred in Ultra-Low Attachment 24-well Plates (3473, Corning) in 100 $\mu$ l of medium, plus $700 \mu 1$ of fresh N2B27 containing 30 $\mathrm{n} \mathrm{ml}^{-1}$ bFGF (PMG0034, Gibco), 5ng ml ${ }^{-1}$ VEGF 165 (PHC9394, Gibco) and 0.5mM L-ascorbic acid phosphate (013-12061, Wako) (N2B27+++) and cultured on an orbital shaker placed at $37^{\circ} \mathrm{C}, 5 \% \mathrm{CO}_{2}$ at $100 \mathrm{rpm}$ (VWR mini shaker). From $120 \mathrm{~h}$ onwards, half medium was changed daily. Unless differently specified, N2B27+++ was applied from 96 to $144 \mathrm{~h}$, while from $144 \mathrm{~h}$ to $168 \mathrm{~h} \mathrm{~N} 2 \mathrm{~B} 27$ was used for medium change.

\section{Live imaging and cell tracking}

Bright-field live imaging of beating gastruloids was performed with a Nikon Ti inverted microscope equipped with an incubation chamber at $37^{\circ} \mathrm{C}, 5 \% \mathrm{CO} 2$. Light-sheet live imaging of Flk1-GFP and Mesp1-GFP gastruloids was performed with a prototype of LS1 live inverted light-sheet microscope (Viventis Microscopy Sarl, Switzerland), at $37^{\circ} \mathrm{C}, 5 \% \mathrm{CO}_{2}$. A volume of $150-200 \mu \mathrm{m}$ was acquired with a $\mathrm{Z}$ spacing of $2-3 \mu \mathrm{m}$ between slices and pictures were captured every $20 \mathrm{~min}$ for Flk1-GFP gastruloids and every $10 \mathrm{~min}$ for tracking of Mesp $1^{+}$cells. 
Flk1-GFP light-sheet video montages were obtained with the Arivis Vision4D software. To track Mesp $1^{+}$cells in Gastruloids from 96 to 120 h, LS1 live light-sheet images were processed with the Fiji Mastodon plugin, using a semi-automatic tracking. Subsequently, Mastodon files were exported for Mamut, and the Fiji Mamut plugin was used to display cell tracks as shown in Figure 1.

\section{Immunofluorescence, confocal and light-sheet imaging on fixed samples}

Immunofluorescence on whole mount gastruloids was performed as previously described (Baillie-Johnson et al., 2015). Briefly, gastruloids were washed in PBS and fixed in 4\% PFA for $2 \mathrm{~h}$ at $4^{\circ} \mathrm{C}$ while shaking. Samples were washed 3 times in PBS and 3 times (10 min each) in blocking buffer (PBS, 10\%FBS, 0,2\%Triton X-100), then blocked for $1 \mathrm{~h}$ at $4{ }^{\circ} \mathrm{C}$ in blocking buffer. Gastruloids were then incubated $\mathrm{O} / \mathrm{N}$ with primary antibodies in blocking buffer, at $4^{\circ} \mathrm{C}$ while shaking. The day after, gastruloids were washed 4 times (20 min each) with blocking buffer, at $4^{\circ} \mathrm{C}$ while shaking, and incubated $\mathrm{O} / \mathrm{N}$ with secondary antibodies and DAPI $\left(2 \mu \mathrm{g} \mathrm{ml} \mathrm{m}^{-}\right.$

${ }^{1}$, Sigma-Aldrich) in blocking buffer, at $4^{\circ} \mathrm{C}$ while shaking. The day after, gastruloids were washed for $1 \mathrm{~h}$ with blocking buffer, at $4^{\circ} \mathrm{C}$ while shaking, then rinsed in PBS, $0,2 \% \mathrm{FBS}, 0,2 \%$ Triton X-100 and mounted on Superfrost plus glass slides (ThermoFisher) with Floromount-G for confocal imaging (Southern Biotech). The following primary antibodies were used: mouse anti-Gata4 (1:500, Santa Cruz Biotechnology, G-4); chicken anti-GFP (1:750, Aves Labs); goat anti-Brachyury (1:300, Santa Cruz Biotechnology, C-19); rat anti-CD31 (1:100, BD, MEC 13.3), mouse anti-cardiac troponin $\mathrm{T}$ (1:100, ThermoFisher, 13-11), rabbit anti-E-Cadherin (1:500, Cell Signaling, 24E10). The following secondary antibodies were used: donkey antichicken 488 AlexaFluor (1:500, Jackson ImmunoResearch); donkey anti-goat AlexaFluor 568 (1:500, ThermoFisher); goat anti-rat AlexaFluor 568 (1:500, ThermoFisher); goat anti-mouse AlexaFluor 647 (1:500, ThermoFisher); donkey anti-rabbit 568 (1:500, ThermoFisher). Confocal pictures were acquired with a Zeiss LSM 700 inverted confocal microscope equipped with a Axiocam MRm black and white camera in the EPFL bioimaging and optics facility. For light-sheet imaging (Fig. 4), samples were mounted in 1\% low-melt agarose and cleared overnight with CUBIC mount solution (Lee et al., 2016). Light-sheet imaging was performed on a Zeiss Light-sheet Z1 microscope equipped with a Plan-Neofluar 20x/1.0 Corr nd=1.45 objective. Light-sheet images were further processed with Imaris software, for 3D rendering and surface generation. 


\section{RNA extraction and qRT-PCR}

RNA was extracted from gastruloids with the RNeasy Micro kit (Qiagen), according to manufacturer's instructions and quantified with a spectrophotometer (ND-1000, Nanodrop). $1 \mu \mathrm{g}$ of RNA was reverse-transcribed with the iScript cDNA Supermix kit (Biorad). cDNA was diluted $1: 10$ and $1.5 \mu l$ of cDNA per reaction were used, in a total volume of $10 \mu 1.384$ well plates were prepared using a robotized liquid handling platform (Hamilton Microlab Star). qPCR was run with a 7900HT Fast PCR machine (Applied Biosystems), using Power SYBR Green PCR Master Mix (Applied Biosystems), with an annealing temperature of $60^{\circ} \mathrm{C}$. Gene expression was normalized on $\beta$-actin expression. Relative fold expression was calculated with the $2-\Delta \Delta \mathrm{CT}$ method. $500 \mathrm{nM}$ of the following primers were used: Mespl FOR GTCTGCAGCGGGGTGTCGTG; Mesp1 REV CGGCGGCGTCCAGGTTTCTA; Nkx2.5 FOR CACATTTTACCCGGGAGCCT; $N k x 2.5$ REV ACCAGATCTTGACCTGCGTG; HCN4 FOR GTGGGGGCCACCTGCTAT; HCN4 REV GTCGGGTGTCAGGCGGGA; $\alpha-$ actinin FOR GGGCTATGAGGAGTGGCTATT; $\quad \alpha$-actinin $\quad$ REV AGTCCTTCTGCAGCAAGATCT; $R y R 2$ FOR TGCATGAGAGCATCAAACGC; $R y R 2$ REV CGCGGAGAGAGGCATTACAT; Tbx5 FOR GGCATGGAAGGAATCAAGGTG; $\begin{array}{llll}\text { Tbx5 REV TTTGGGATTAAGGCCAGTCAC; } & \text { Tbx1 FOR }\end{array}$ CTGTGGGACGAGTtCAATCAG; Tbxl REV TTGTCATCTACGGGCACAAAG; Isll FOR ATGATGGTGGTTTACAGGCTAAC; Isll REV TCGATGCTACTTCACTGCCAG; FGF10 FOR TCAGCGGGACCAAGAATGAAG; FGF10 REV CGGCAACAACTCCGATTTCC; $\beta$-actin FOR CTGTCGAGTCGCGTCCACC; $\beta$-actin REV CGCAGCGATATCGTCATCCA.

\section{RNAscope}

Gastruloids were washed in PBS and fixed $\mathrm{O} / \mathrm{N}$ in $4 \% \mathrm{PFA}$, at $4{ }^{\circ} \mathrm{C}$ while shaking. The day after, samples were washed 3 times in PBS and included in HistoGel (ThermoFisher) blocks. HistoGel blocks were then processed with a Tissue-Tek VIP 6 AI Vacuum Infiltration Processor (Sakura) and included in paraffin. Paraffin blocks were cut at $4 \mu \mathrm{m}$ with a Hyrax M25 microtome (Zeiss). RNA-scope was performed with the ACDBio Manual assay kit using RNAscope Probe-Mm-Tbx1 (481911), RNAscope Probe-Mm-Is11-C3 (451931-C3) and RNAscope Probe-Mm-Tbx5-C2 (519581-C2) probes, according to manufacturer's instructions. Polr2a-C1, Ppib-C2 and Ubiquitin-C3 probes were used as positive and negative controls. 
Pictures were acquired with an upright Leica DM5500 microscope equipped with a CCD DFC 3000 black and white camera.

\section{Flow cytometry analysis and FACS}

Gastruloids were collected, washed in PBS, and digested in $4 \mathrm{mg} \mathrm{ml}^{-1}$ dispase I (Roche), $3 \mathrm{mg}$ $\mathrm{ml}^{-1}$ collagenase IV (Gibco) and $100 \mu \mathrm{g} \mathrm{ml}^{-1}$ DNase I (Roche) in PBS (2 digestion cycles at $37^{\circ} \mathrm{C}$, 5 min each; gentle pipetting was applied between the two cycles to mechanically dissociate the gastruloids). Digestion was blocked with DMEM containing 10\% FBS, then samples were centrifuged and the cell pellet was resuspended in sorting buffer (PBS, 5\%FBS, $1 \mathrm{mM}$ EDTA, $1 \% \mathrm{P} / \mathrm{S}$ ) for antibody staining. Samples were incubated for $1 \mathrm{~h}$ on ice with antibodies, and 30min on ice with Aqua live/dead fixable dead cell stain kit $(405 / 525 \mathrm{~nm}$, Invitrogen) or 10 min on ice with DAPI. Unstained, FMO and single color samples were used as controls. The following antibodies were used: anti CD31-PE 1:1200 (BD, MEC 13.3), anti VEGFR2/Flk1-APC 1:200 (Biolegend, Avas12); anti CXCR4-APC 1:100 (BD, 2B11). Samples were analyzed with a BD LSR II flow cytometer. Cell sorting was performed using a BD FACSAria Fusion cell sorter.

\section{In vitro angiogenesis assay}

168-h Flk1-GFP gastruloids were collected and digested as described above for FACS analysis. $F l k I^{+}$and $F l k l^{-}$cells were isolated through cell sorting using a BD FACSAria Fusion cell sorter. $5 * 10^{4}$ cells per condition were plated in IBIDI $\mu$-angiogenesis slides pre-coated with $10 \mu 1$ reduced growth factor Matrigel (Corning) in the lower chamber. Undifferentiated mESCs and HUVEC were used as negative and positive controls, respectively. Live imaging of tube formation was performed with a Nikon Ti inverted microscope equipped with an incubation chamber at $37^{\circ} \mathrm{C}, 5 \% \mathrm{CO}_{2}$, with acquisitions every $15 \mathrm{~min}$.

\section{Calcium imaging}

To image calcium fluxes, gastruloids were incubated for $1 \mathrm{~h}$ with $8 \mu \mathrm{M}$ Cal-520 (AAT Bioquest) at $37^{\circ} \mathrm{C}, 5 \% \mathrm{CO}_{2}$. Gastruloids were then transferred to fresh medium before imaging. Imaging was performed with a Light-sheet Z1 microscope (Zeiss) equipped with an environmental chamber to maintain gastruloids at $37^{\circ} \mathrm{C}$ and $5 \% \mathrm{CO}_{2}$. For imaging, gastruloids were embedded in $1 \%$ low melt agarose and the chamber was filled with culture medium. Nifidepine (Sigma Aldrich, $10 \mu \mathrm{M}$ ) and isoproterenol (isoprenaline hydrochloride, Sigma I5627, $1 \mu \mathrm{M}$ ) were added 
with a syringe directly to the imaging chamber during acquisition. The analysis of calcium spikes was performed with the Fiji Stacks-plot Z-Axis profile plugin. The baseline intensity was normalized to the minimum value over $10 \mathrm{sec}$. The ratio of fluorescence intensity to baseline intensity was calculated and results are shown as the percentage of increase over the baseline, which shows the relative changes in intracellular $\mathrm{Ca}^{2+}$.

\section{Crescent analysis}

Analysis of crescent geometrical properties was performed using a custom-made Matlab script for Imaris files with a Imaris XT feature and EasyXT. Initially, we generated artificial shapes to be used as reference for defined geometrical metrics. Using a custom script (crescent_generator.m), 3D objects were generated to mimic crescent structures with different characteristics. To do so, the script creates two spot objects, makes channels from these spots and finally subtracts one channel to the other. To analyze surfaces, 3D images from non-beating organoids at $144 \mathrm{~h}$ and beating organoids at $168 \mathrm{~h}$ were acquired with a Light-sheet $\mathrm{Z} 1$ microscope (Zeiss) after clearing, as described above. For each individual stack, a surface was created using a dedicated user interface (GUI_DetectAndAnalyze.m), defining the object that should be created for each channel (settings_.m). Due to variability in background and signal intensity, the threshold (absolute intensity) was adjusted manually for each surface. Using a custom script (makeCroissantMeasure final.m) geometrical measurements were computed and the results exported in csv table. In the graph, we plot the measure of Spareness, which is described as the ratio between the volume of the object and the volume of the best fitted ellipsoid. All scripts and settings used for analysis are available at go.epfl.ch/Crescent_Analysis.

\section{Statistics}

All data shown in column graphs are expressed as mean $\pm \mathrm{SD}$, apart from the graph showing Calcium spikes frequency, which is expressed as mean \pm whiskers from min to max. All other graphs show single data points. Statistical analysis between two columns was performed using two-tailed unpaired Student's t test, whereas data containing more than two experimental groups were analyzed with one-way analysis of variance followed by Bonferroni's test. To calculate the significance of the percentage of increase over baseline frequency after Isoproterenol administration, we applied a one sample $t$ test. Statistical significance was calculated using the Graphpad Prism software, that was also used to generate all graphs. ${ }^{*} \mathrm{P}<0.05 ; * * \mathrm{P}<0.01 ; * * * \mathrm{P}<0.001$; confidence intervals 95\%; alpha level 0.05 . 


\section{Supplemental Figures}

A
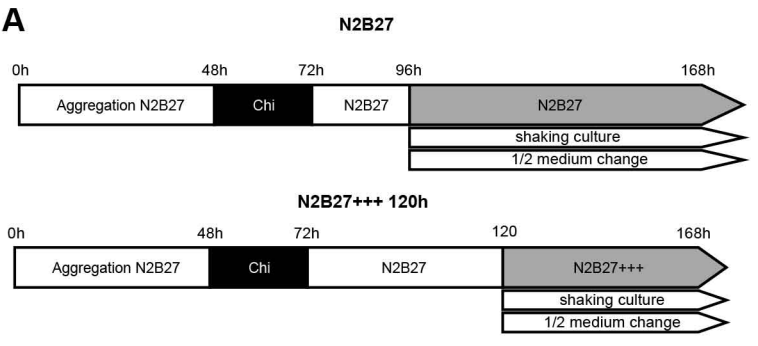

B

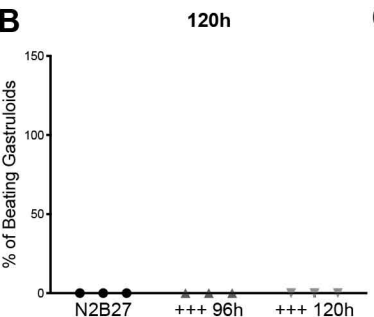

$\mathbf{F}$

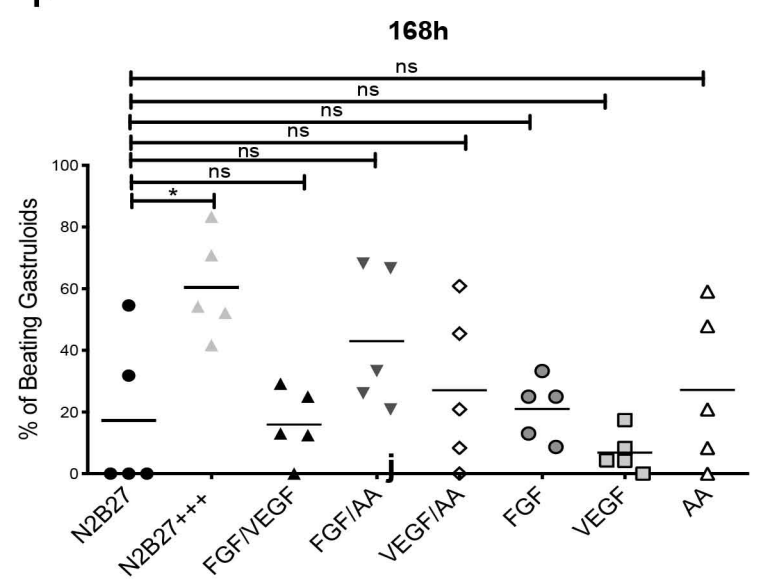

C

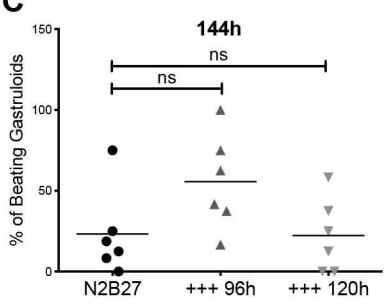

G

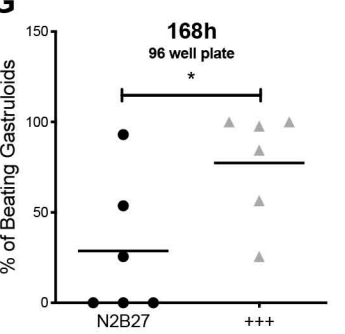

I

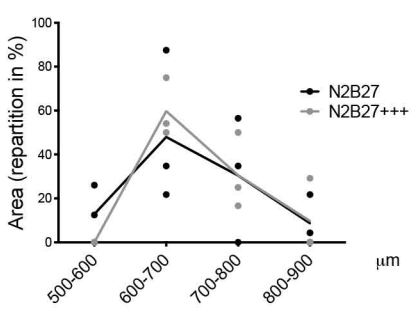

E

D
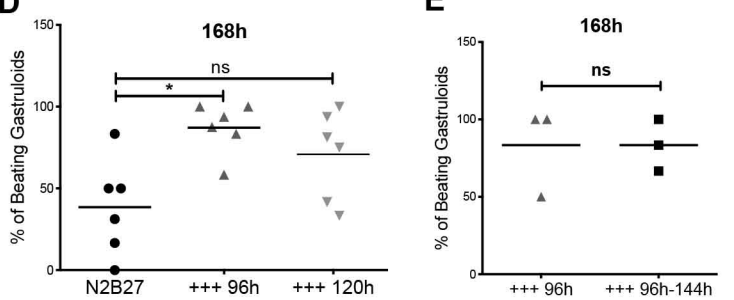

H

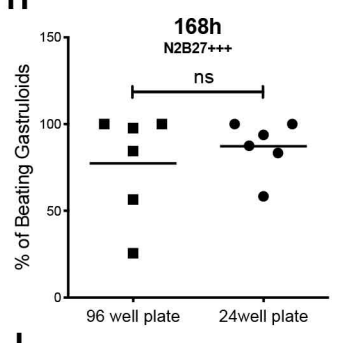

$\mathrm{J}$

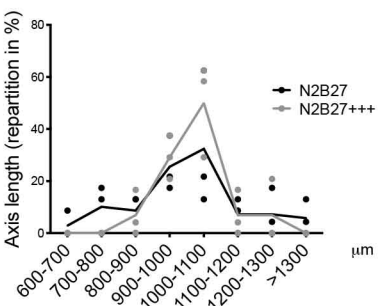

Figure S1 | Comparison between N2B27 and N2B27+++ culture conditions. A, Schematics of the different culture conditions tested. B-E, Percentage of beating gastruloids from 120 to $168 \mathrm{~h}$ in the different conditions. F, Exposure to N2B27+++ induces beating at higher frequencies compared to exposure to single factors or couples. G, H Frequencies of beating gastruloids grown in 96 well plates $(\mathbf{G})$ and comparison with those grown in 24 well plates from 144h (H). Each dot in B-H represent an independent experiment. I-J, quantification of area and axis at $168 \mathrm{~h}$ of gastruloids grown in N2B27 or N2B27+++. Mean of $n=3$ independent experiments. 
bioRxiv preprint doi: https://doi.org/10.1101/802181; this version posted October 17, 2019. The copyright holder for this preprint (which was not certified by peer review) is the author/funder, who has granted bioRxiv a license to display the preprint in perpetuity. It is made available under aCC-BY-NC-ND 4.0 International license.

A

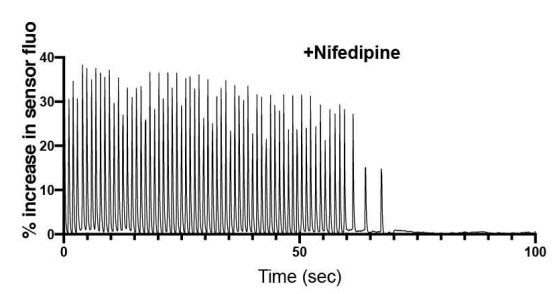

D

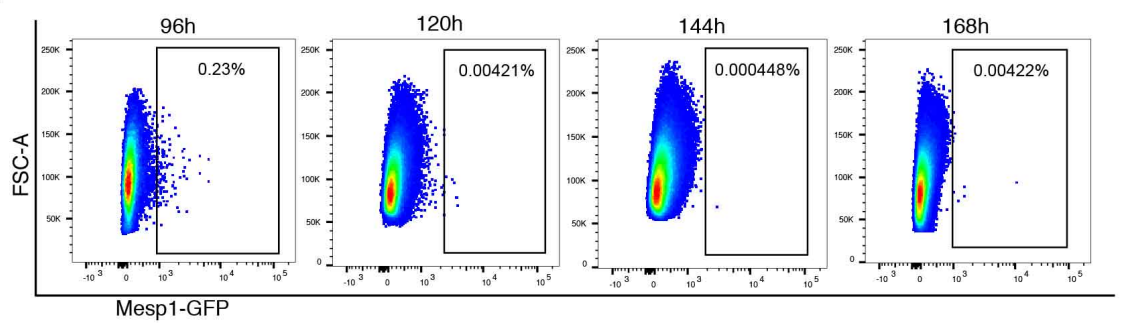

$\mathbf{F}$

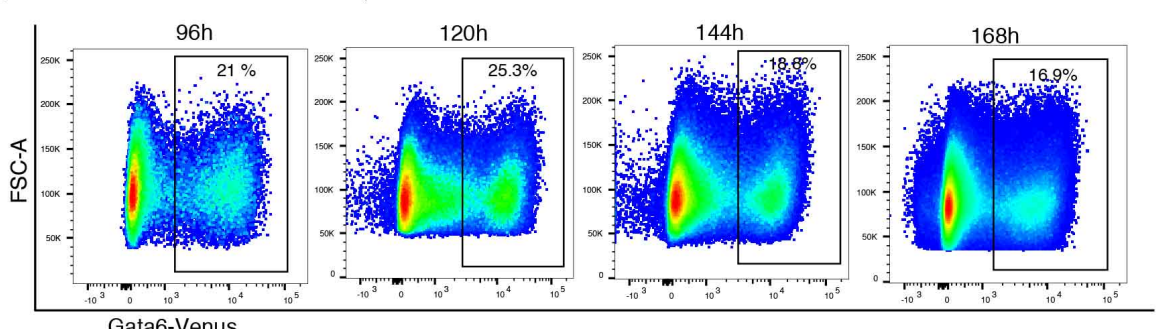

C
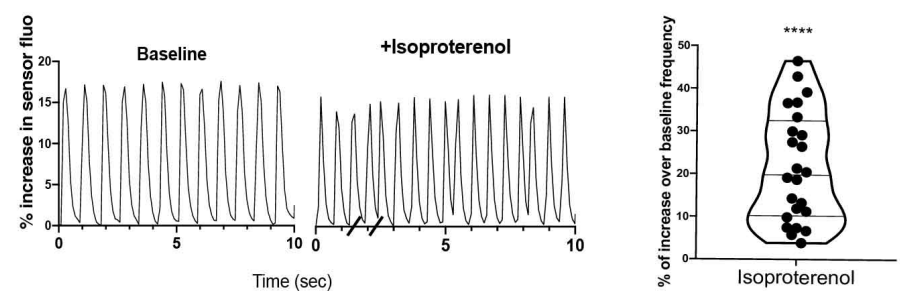

E

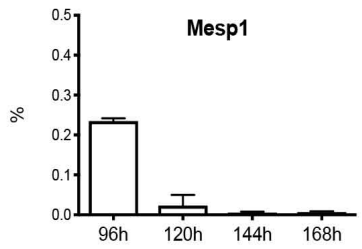

G

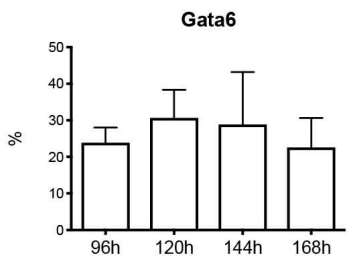

Figure S2 | Development of the cardiac portion of gastruloids. A-C, Treatment of $168 \mathrm{~h}$ gastruloids with Nifedipine ( $n=9$ gastruloids) (A) or Isoproterenol (B, C) abolish or fastens calcium spiking in the gastruloid cardiac portion, respectively. Graph in (B) shows representative calcium spikes of gastruloids before and after Isoproterenol treatment, and graph in $(\mathbf{C})$ the percentage of increase over baseline frequency of gastruloids after Isoproterenol treatment. $n=24$ gastruloids. D, E, FACS analysis of Mesp1-GFP gastruloids from 96 to 168 (D) and relative quantification (E). $n=2$ independent experiments. F, G, FACS analysis of Gata6-Venus gastruloids from 96 to $168(\mathbf{F})$ and relative quantification $(\mathbf{G}) . n=2$ independent experiments. 
bioRxiv preprint doi: https://doi.org/10.1101/802181; this version posted October 17,2019 . The copyright holder for this preprint (which was not certified by peer review) is the author/funder, who has granted bioRxiv a license to display the preprint in perpetuity. It is made available under aCC-BY-NC-ND 4.0 International license.

A

$96 \mathrm{~h}$

$120 \mathrm{~h}$

$144 \mathrm{~h}$

$68 \mathrm{~h}$
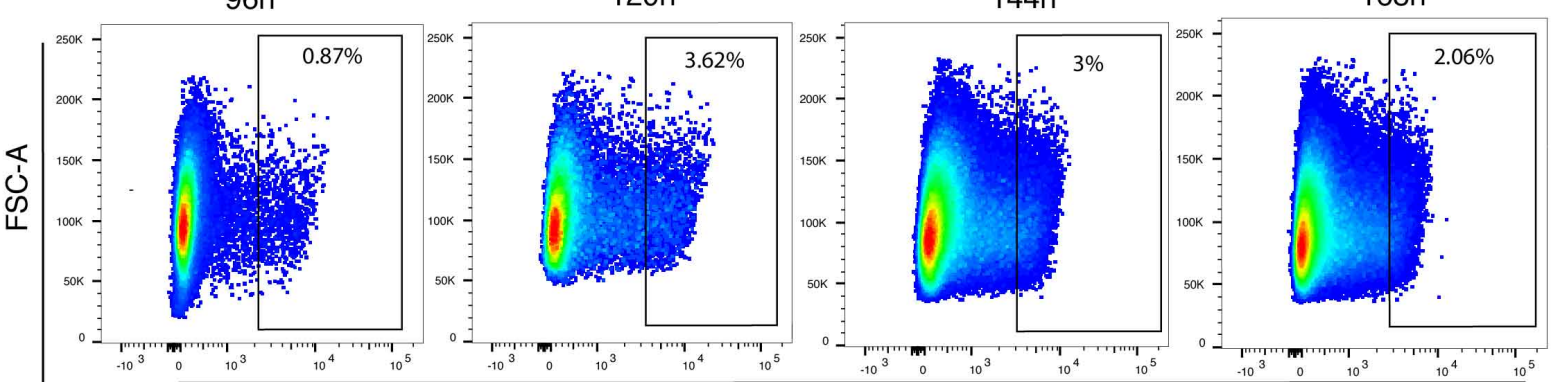

Flk1-GFP

B

C

$168 \mathrm{~h}$

$168 \mathrm{~h}$

$168 \mathrm{~h}$
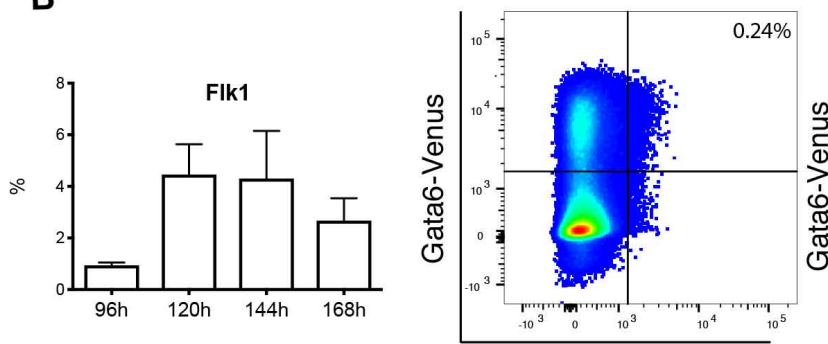

Flk1
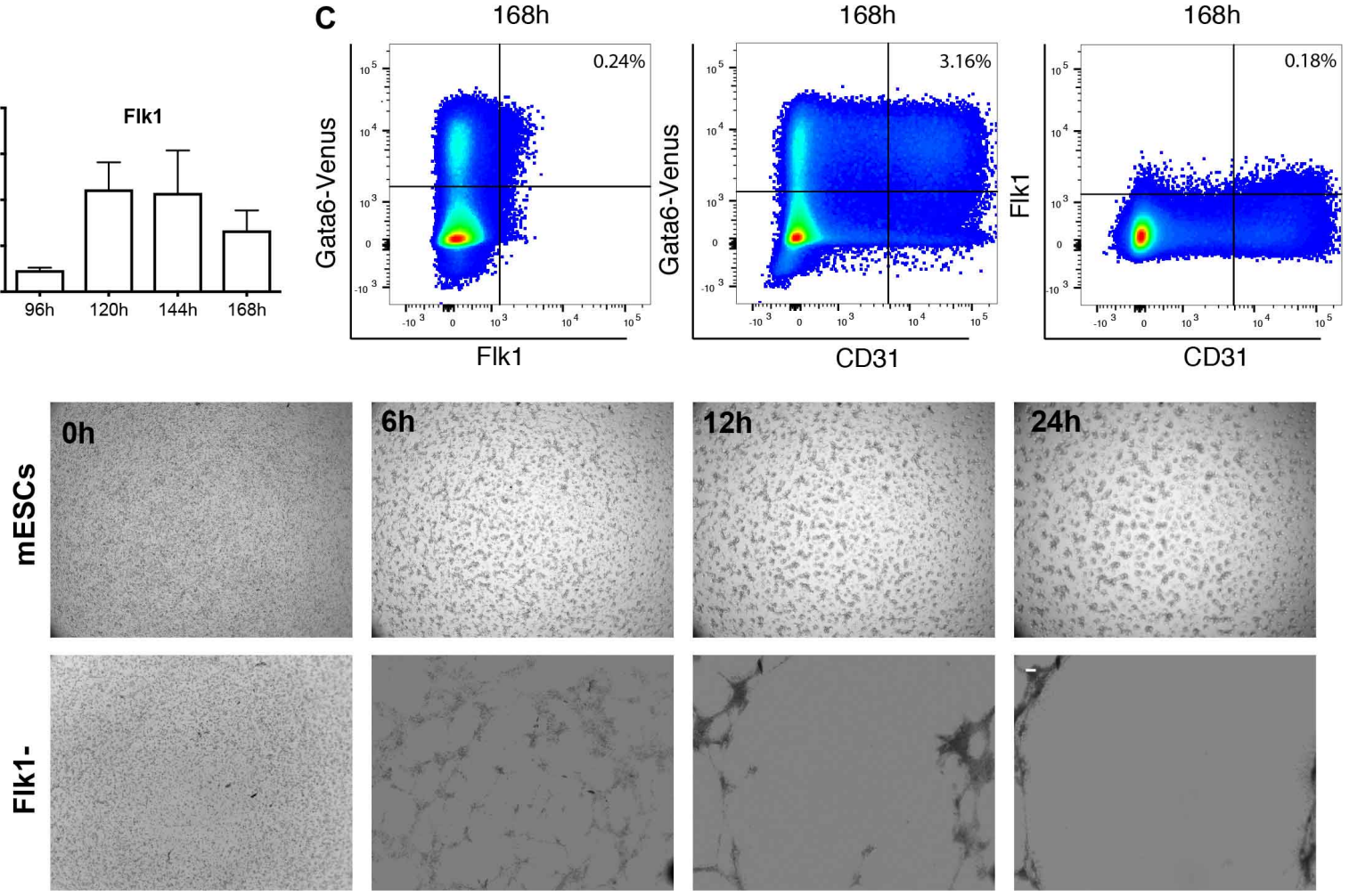

Figure S3 | Flk1 marks a vascular-like compartment. A, B FACS analysis of Flk1-GFP gastruloids from 96 to 168 (A) and relative quantification (B). $n=2$ independent experiments. C, FACS analysis of Gata6-Venus gastruloids showing co-expression of Flk1 and CD31. $n=2$ independent experiments. D, angiogenesis assay showing inability to form vascular-like tubes by undifferentiated mESCs and Flk1' cells. Scale bar, $100 \mu \mathrm{m}$. 
bioRxiv preprint doi: https://doi.org/10.1101/802181; this version posted October 17, 2019. The copyright holder for this preprint (which was not certified by peer review) is the author/funder, who has granted bioRxiv a license to display the preprint in perpetuity. It is made available under aCC-BY-NC-ND 4.0 International license.

\section{Supplemental Movie Legends}

Supplemental Movie 1 | Example of a non-beating gastruloid cultured in N2B27 and a beating gastruloid cultured in $\mathrm{N} 2 \mathrm{~B} 27+++$

Supplemental Movie 2 | Calcium imaging of a gastruloid beating portion with Cal520

Supplemental Movie $3 \mid M_{\text {Mesp }}^{+}$light-sheet cell tracking and 3D rendering of Mesp $^{+}$ cell tracking from 96 to $120 \mathrm{~h}$

Supplemental Movie 4 | Development of a $\mathrm{FlkI}^{+}$network in embryonic organoids. Rendering of Light-sheet LS1 live imaging of the anterior portion of a Flk1-GFP gastruloid from 120 to $144 \mathrm{~h}$. The video was created and edited with the Arivis Vision4D software.

Supplemental Movie 5 | Localization of the gut tube, endocardium and cardiac portion in embryonic organoids. Rendering of the immunostaining in Figure $4 \mathbf{D}, \mathbf{E}$ showing respective localization of the gut-like tube, the cardiac portion and the endocardial-like layer in 3D. Imaging was performed using Light-sheet Z1 microscope on optically cleared samples. The video was created and edited with Imaris software. 


\section{References}

Andersen, P., Tampakakis, E., Jimenez, D.V., Kannan, S., Miyamoto, M., Shin, H.K., Saberi, A., Murphy, S., Sulistio, E., Chelko, S.P., et al. (2018). Precardiac organoids form two heart fields via Bmp/Wnt signaling. Nat. Commun. 9, 3140.

Baillie-Johnson, P., van den Brink, S.C., Balayo, T., Turner, D.A., and Martinez Arias, A. (2015). Generation of Aggregates of Mouse Embryonic Stem Cells that Show Symmetry Breaking, Polarization and Emergent Collective Behaviour In Vitro. J. Vis. Exp. JoVE.

Beccari, L., Moris, N., Girgin, M., Turner, D.A., Baillie-Johnson, P., Cossy, A.-C., Lutolf, M.P., Duboule, D., and Arias, A.M. (2018). Multi-axial self-organization properties of mouse embryonic stem cells into gastruloids. Nature 562, 272-276.

Bondue, A., Tännler, S., Chiapparo, G., Chabab, S., Ramialison, M., Paulissen, C., Beck, B., Harvey, R., and Blanpain, C. (2011). Defining the earliest step of cardiovascular progenitor specification during embryonic stem cell differentiation. J. Cell Biol. 192, 751-765.

van den Brink, S.C., Baillie-Johnson, P., Balayo, T., Hadjantonakis, A.-K., Nowotschin, S., Turner, D.A., and Martinez Arias, A. (2014). Symmetry breaking, germ layer specification and axial organisation in aggregates of mouse embryonic stem cells. Dev. Camb. Engl. 141, 4231-4242.

Brutsaert, D.L. (2003). Cardiac endothelial-myocardial signaling: its role in cardiac growth, contractile performance, and rhythmicity. Physiol. Rev. 83, 59-115.

Brutsaert, D.L., Fransen, P., Andries, L.J., De Keulenaer, G.W., and Sys, S.U. (1998).

Cardiac endothelium and myocardial function. Cardiovasc. Res. 38, 281-290.

Clevers, H. (2016). Modeling Development and Disease with Organoids. Cell 165, 15861597.

Cortes, Francou Alexandre, De Bono Christopher, and Kelly Robert G. (2018). Epithelial Properties of the Second Heart Field. Circ. Res. 122, 142-154.

Deluz, C., Friman, E.T., Strebinger, D., Benke, A., Raccaud, M., Callegari, A., Leleu, M., Manley, S., and Suter, D.M. (2016). A role for mitotic bookmarking of SOX2 in pluripotency and differentiation. Genes Dev. 30, 2538-2550.

Freyer, L., Schröter, C., Saiz, N., Schrode, N., Nowotschin, S., Martinez-Arias, A., and Hadjantonakis, A.-K. (2015). A loss-of-function and H2B-Venus transcriptional reporter allele for Gata6 in mice. BMC Dev. Biol. 15, 38.

Giacomelli, E., Bellin, M., Sala, L., Meer, B.J. van, Tertoolen, L.G.J., Orlova, V.V., and Mummery, C.L. (2017). Three-dimensional cardiac microtissues composed of cardiomyocytes and endothelial cells co-differentiated from human pluripotent stem cells. Development dev.143438.

Harrison, S.E., Sozen, B., Christodoulou, N., Kyprianou, C., and Zernicka-Goetz, M. (2017). Assembly of embryonic and extraembryonic stem cells to mimic embryogenesis in vitro. Science 356. 
Harvey, R.P. (2002). Patterning the vertebrate heart. Nat. Rev. Genet. 3, 544-556.

Hosseini, H.S., Garcia, K.E., and Taber, L.A. (2017). A new hypothesis for foregut and heart tube formation based on differential growth and actomyosin contraction. Dev. Camb. Engl. 144, 2381-2391.

Huebsch, N., Loskill, P., Deveshwar, N., Spencer, C.I., Judge, L.M., Mandegar, M.A., Fox, C.B., Mohamed, T.M.A., Ma, Z., Mathur, A., et al. (2016). Miniaturized iPS-Cell-Derived Cardiac Muscles for Physiologically Relevant Drug Response Analyses. Sci. Rep. 6, 24726.

Ivanovitch, K., Temiño, S., and Torres, M. (2017). Live imaging of heart tube development in mouse reveals alternating phases of cardiac differentiation and morphogenesis.

Jakobsson, L., Franco, C.A., Bentley, K., Collins, R.T., Ponsioen, B., Aspalter, I.M., Rosewell, I., Busse, M., Thurston, G., Medvinsky, A., et al. (2010). Endothelial cells dynamically compete for the tip cell position during angiogenic sprouting. Nat. Cell Biol. 12, 943-953.

Kattman, S.J., Huber, T.L., and Keller, G.M. (2006). Multipotent flk-1+ cardiovascular progenitor cells give rise to the cardiomyocyte, endothelial, and vascular smooth muscle lineages. Dev. Cell 11, 723-732.

Kelly, R.G., Buckingham, M.E., and Moorman, A.F. (2014). Heart Fields and Cardiac Morphogenesis. Cold Spring Harb. Perspect. Med. 4, a015750.

Kidokoro, H., Yonei-Tamura, S., Tamura, K., Schoenwolf, G.C., and Saijoh, Y. (2018). The heart tube forms and elongates through dynamic cell rearrangement coordinated with foregut extension. Dev. Camb. Engl. 145.

Lancaster, M.A., and Knoblich, J.A. (2014). Organogenesis in a dish: Modeling development and disease using organoid technologies. Science 345, 1247125.

Le Garrec, J.-F., Domínguez, J.N., Desgrange, A., Ivanovitch, K.D., Raphaël, E., Bangham, J.A., Torres, M., Coen, E., Mohun, T.J., and Meilhac, S.M. A predictive model of asymmetric morphogenesis from 3D reconstructions of mouse heart looping dynamics. ELife 6.

Lee, E., Choi, J., Jo, Y., Kim, J.Y., Jang, Y.J., Lee, H.M., Kim, S.Y., Lee, H.-J., Cho, K., Jung, N., et al. (2016). ACT-PRESTO: Rapid and consistent tissue clearing and labeling method for 3-dimensional (3D) imaging. Sci. Rep. 6, 18631.

Lescroart, F., Chabab, S., Lin, X., Rulands, S., Paulissen, C., Rodolosse, A., Auer, H., Achouri, Y., Dubois, C., Bondue, A., et al. (2014). Early lineage restriction in temporally distinct populations of Mesp1 progenitors during mammalian heart development. Nat. Cell Biol. 16, 829-840.

Lind, J.U., Busbee, T.A., Valentine, A.D., Pasqualini, F.S., Yuan, H., Yadid, M., Park, S.-J., Kotikian, A., Nesmith, A.P., Campbell, P.H., et al. (2017). Instrumented cardiac microphysiological devices via multimaterial three-dimensional printing. Nat. Mater. 16, 303-308.

Lough, J., and Sugi, Y. (2000). Endoderm and heart development. Dev. Dyn. 217, 327-342. 
Ma, Z., Wang, J., Loskill, P., Huebsch, N., Koo, S., Svedlund, F.L., Marks, N.C., Hua, E.W., Grigoropoulos, C.P., Conklin, B.R., et al. (2015). Self-organizing human cardiac microchambers mediated by geometric confinement. Nat. Commun. 6, 7413.

Mills, R.J., Parker, B.L., Quaife-Ryan, G.A., Voges, H.K., Needham, E.J., Bornot, A., Ding, M., Andersson, H., Polla, M., Elliott, D.A., et al. (2019). Drug Screening in Human PSCCardiac Organoids Identifies Pro-proliferative Compounds Acting via the Mevalonate Pathway. Cell Stem Cell 0.

Miquerol, L., and Kelly, R.G. (2013). Organogenesis of the vertebrate heart. Wiley Interdiscip. Rev. Dev. Biol. 2, 17-29.

Narmoneva, Vukmirovic Rada, Davis Michael E., Kamm Roger D., and Lee Richard T. (2004). Endothelial Cells Promote Cardiac Myocyte Survival and Spatial Reorganization. Circulation 110, 962-968.

Nascone, N., and Mercola, M. (1995). An inductive role for the endoderm in Xenopus cardiogenesis. Development 121, 515-523.

Polonchuk, L., Chabria, M., Badi, L., Hoflack, J.-C., Figtree, G., Davies, M.J., and Gentile, C. (2017). Cardiac spheroids as promising in vitro models to study the human heart microenvironment. Sci. Rep. 7, 7005.

Rajala, K., Pekkanen-Mattila, M., and Aalto-Setälä, K. (2011). Cardiac Differentiation of Pluripotent Stem Cells. Stem Cells Int. 2011.

Rivron, N.C., Frias-Aldeguer, J., Vrij, E.J., Boisset, J.-C., Korving, J., Vivié, J., Truckenmüller, R.K., Oudenaarden, A., Blitterswijk, C.A., and Geijsen, N. (2018). Blastocyst-like structures generated solely from stem cells. Nature 557, 106-111.

Rossi, G., Manfrin, A., and Lutolf, M.P. (2018). Progress and potential in organoid research. Nat. Rev. Genet. 19, 671.

Saga, Y., Hata, N., Kobayashi, S., Magnuson, T., Seldin, M.F., and Taketo, M.M. (1996). MesP1: a novel basic helix-loop-helix protein expressed in the nascent mesodermal cells during mouse gastrulation. Dev. Camb. Engl. 122, 2769-2778.

Sasai, Y. (2013). Cytosystems dynamics in self-organization of tissue architecture. Nature 493, 318-326.

Schultheiss, T.M., Xydas, S., and Lassar, A.B. (1995). Induction of avian cardiac myogenesis by anterior endoderm. Development 121, 4203-4214.

Shao, Y., Taniguchi, K., Townshend, R.F., Miki, T., Gumucio, D.L., and Fu, J. (2017a). A pluripotent stem cell-based model for post-implantation human amniotic sac development. Nat. Commun. 8, 208.

Shao, Y., Taniguchi, K., Gurdziel, K., Townshend, R.F., Xue, X., Yong, K.M.A., Sang, J., Spence, J.R., Gumucio, D.L., and Fu, J. (2017b). Self-organized amniogenesis by human pluripotent stem cells in a biomimetic implantation-like niche. Nat. Mater. 16, 419-425. 
Sozen, B., Amadei, G., Cox, A., Wang, R., Na, E., Czukiewska, S., Chappell, L., Voet, T., Michel, G., Jing, N., et al. (2018). Self-assembly of embryonic and two extra-embryonic stem cell types into gastrulating embryo-like structures. Nat. Cell Biol. 20, 979.

Tyser, R.C., Miranda, A.M., Chen, C.-M., Davidson, S.M., Srinivas, S., and Riley, P.R. (2016). Calcium handling precedes cardiac differentiation to initiate the first heartbeat. ELife 5 .

Varner, V.D., and Taber, L.A. (2012). Not just inductive: a crucial mechanical role for the endoderm during heart tube assembly. Dev. Camb. Engl. 139, 1680-1690.

Zhao, Y., Rafatian, N., Feric, N.T., Cox, B.J., Aschar-Sobbi, R., Wang, E.Y., Aggarwal, P., Zhang, B., Conant, G., Ronaldson-Bouchard, K., et al. (2019). A Platform for Generation of Chamber-Specific Cardiac Tissues and Disease Modeling. Cell 176, 913-927.e18.

Zheng, Y., Xue, X., Shao, Y., Wang, S., Esfahani, S.N., Li, Z., Muncie, J.M., Lakins, J.N., Weaver, V.M., Gumucio, D.L., et al. (2019). Controlled modelling of human epiblast and amnion development using stem cells. Nature 573, 421-425. 